\title{
Research Article \\ Power Distribution and Possible Influence on Fuel Failure in WWER-1000
}

\begin{abstract}
Ján Mikuš
Research Centre Řež Ltd., 25068 Řež, Czech Republic

Correspondence should be addressed to Ján Mikuš, mik@ujv.cz

Received 21 March 2008; Revised 14 August 2008; Accepted 22 August 2008

Recommended by Igor Jencic

The work is focused on the influence of investigation of some core heterogeneities and construction materials on the space power (fission rate) distribution in WWER-1000-type cores, especially from viewpoint of the values and gradient occurrence that could result in static loads with some consequences, for example, fuel pin (FP) or fuel assembly (FA) bowing and possible contribution to the FP failure root causes. For this purpose, experimental data and their analysis from two earlier performed measurements on light water, zero-power reactor LR-0 were used, concerning the relative radial power distribution determined by measurements in a WWER-1000-type core containing single FPs with homogeneous gadolinium admixture $\left(\mathrm{Gd}_{2} \mathrm{O}_{3}\right)$ and the relative radial power distribution determined by measurements in FA situated on the periphery of a WWER-1000-type core neighbouring the baffle (thermal shielding).
\end{abstract}

Copyright (c) 2008 Ján Mikuš. This is an open access article distributed under the Creative Commons Attribution License, which permits unrestricted use, distribution, and reproduction in any medium, provided the original work is properly cited.

\section{INTRODUCTION}

The LR-0 reactor in the Nuclear Research Institute Řež plc is an experimental facility for determination of the neutronphysical characteristics of the WWER- and PWR-type lattices and shielding with $\mathrm{UO}_{2}$ or MOX fuel. Exploitation of this facility is determined by maximum power of $5 \mathrm{~kW}$ and maximum thermal neutron flux density of $10^{13} \mathrm{~m}^{-2} \mathrm{~s}^{-1}$, atmospheric pressure, and room temperature (or heating up to $70^{\circ} \mathrm{C}$ ). The fuel consists of the shortened WWER-1000and WWER-440-type fuel assemblies (FAs) containing the fuel pins (FPs) with $\mathrm{UO}_{2}$ pellets (O.D. $7.53 \mathrm{~mm}$, internal central hole $1.4 \mathrm{~mm}$ ) having a $\mathrm{Zr}+1 \% \mathrm{Nb}$ cladding (O.D. $9.15 \times 0.72 \mathrm{~mm}$ ) with the active length of $1250 \mathrm{~mm}$ and enrichment of $1.6-4.4 \mathrm{wt} . \%$ in ${ }^{235} \mathrm{U}$. The FAs can be arranged in a reactor vessel of aluminium (diameter $3.5 \mathrm{~m}$, height $6.5 \mathrm{~m}$ ); the criticality is controlled by moderator level (boron acid with concentration up to $12 \mathrm{~g} / \mathrm{L}$ ) and control clusters ( $\mathrm{B}_{4} \mathrm{C}$ pellets).

The most important applications on LR-0 reactor concern the nuclear safety and economy of the WWER-type nuclear power plants (NPPs) operation. In the frame of their modernization, a set of experiments has been performed on the LR-0 reactor, for example, with new type FAs with various burnable absorbers arranged in appropriate configurations and cores of the both WWER-440 and WWER-1000 types including a number of experiments with FAs containing single FPs with Gd (Gd FPs) burnable absorber $\left(\mathrm{Gd}_{2} \mathrm{O}_{3}\right)$ integrated into fuel (e.g., $\left.[1,2]\right)$. Next experiments concerned the reactor pressure vessel dosimetry and they included measurements of the power (fission rate) distribution in periphery FAs neighbouring the baffle in WWER-1000-type cores (e.g., [3]). Results of power (fission rate) distribution measurements presented in [1-3] are used and analysed in this work.

It is well known that presence of single Gd FPs in reactor core results in a local depression of thermal neutrons in these FPs and corresponding gradients in neighbouring FPs. As for the baffle influence, thermal neutron gradients can be expected in neighbouring FAs, too. Similar situation can also be stated as for the power release and corresponding temperature.

It is also well known (e.g., $[4,5])$ that neutron flux nonuniformity, gradients of the temperature, and neutron current can represent root causes of the FP/FA growth and bowing leading to local limitation of coolant flow, reduction of heat transfer, magnifying cladding corrosion, and pellet/cladding interaction (PCI). Therefore detailed 

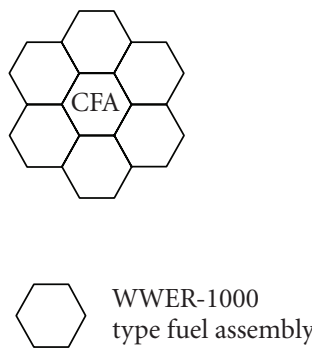

(a)

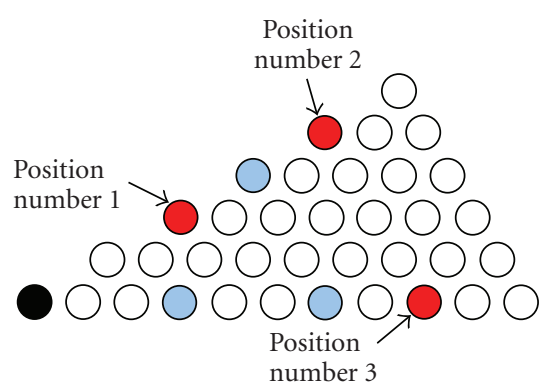

$\bigcirc$ Fuel pin, with Gd
Central tube
Guide tube

(b)

FIGURE 1: (a) Schematic arrangement of the LR-0 reactor core containing 7 WWER-1000 type-FAs with central FA (CFA) for Gd FP influence investigation and (b) the 30 -degree symmetry sector of the CFA with 3 Gd FPs.

information about power distribution in FA containing single Gd FPs and in periphery FA neighbouring the baffle in WWER-1000-type core can be useful for above phenomenon investigation. Since such data cannot be obtained in the NPPs, some experiments on research reactors are provided.

As for the fuel performance reliability and fuel failure rates and root causes concerning WWER fuel, it was stated [6] that an overview on WWER fuel failure root causes identified during 1992-2002 shows that

(i) besides a large number of unidentified causes, the most frequent cause is damage by debris;

(ii) different from Western PWR fuel experience, fuelrod-to-spacer-fretting does not play a significant role;

(iii) at the same time there is a very high number of defected fuel where no failure cause is known.

In case of the PWR fuel the EPRI evaluation of 2004 on US PWR fuel failure rates shows [6] that

(i) after a continuous decrease from 1980 to 2001, there is some increase observed in 2002 and 2003;

(ii) the major contributor to fuel failure rates in PWRs remains grid-to-rod fretting;

(iii) there is also an increase of fuel failures with unknown root causes that primarily affects optimised fuel designs with a thinner rod diameter.

The above information is in accordance with results concerning examination of 5 WWER-440 and 7 WWER1000 FAs presented in [7]: causes of failure are debris fretting (54\%), local overheating (15\%), grid-rod fretting ( $8 \%)$, and "the cause is not determined" (23\%).

\section{AIM OF WORK}

The aim of this work is providing some information about below heterogeneities and construction material influence on radial power (fission rate) distribution in WWER-1000-type cores, namely, concerning the

(i) single Gd FPs by means of power release in an FA containing these FPs,

(ii) baffle by means of power release in selected FPs in neighbouring FA.

\section{INFLUENCE OF SINGLE FUEL PINS WITH GADOLINIUM ON POWER DISTRIBUTION IN A WWER-1000-TYPE CORE}

\subsection{Experimental arrangement and conditions}

The materials published in $[1,2]$ were used for this work preparation. Experiment was realised on reactor LR-0 with critical height of the $\mathrm{H}_{2} \mathrm{O}$ moderator level being $266.10 \mathrm{~mm}$ ("0" level is at the lower end of FP active part), at atmospheric pressure and room temperature in a WWER1000-type core shown in Figure 1(a), consisting of 7 FAs, each containing 312 FPs with $4.4 \%$ enrichment, whereas $18 \mathrm{FPs}$ in central FA (CFA) were replaced by FPs with $3.6 \%$ enrichment containing $2.0 \%$ (by weight) of $\mathrm{Gd}_{2} \mathrm{O}_{3}$ admixture (Gd FP pellets with O.D. $7.50 \mathrm{~mm}$, internal central hole $1.5 \mathrm{~mm}$ ), arranged with FP pitch of $12.75 \mathrm{~mm}$; the 30 degree symmetry sector of CFA with $3 \mathrm{Gd}$ FPs is shown in Figure 1(b). More information concerning experimental arrangement and conditions can be found in $[1,2]$.

\subsection{Results}

The power (fission rate) distribution was determined by means of gamma scanning of irradiated FPs, measuring their gamma radiation in the energy range of 600 to $900 \mathrm{keV}$. The radial distribution (in a plane perpendicular to the core axis) was determined measuring FPs activity of $50 \mathrm{~mm}$ region of FP surface at the central irradiated part along rotating FP using corresponding collimator. Measurements 


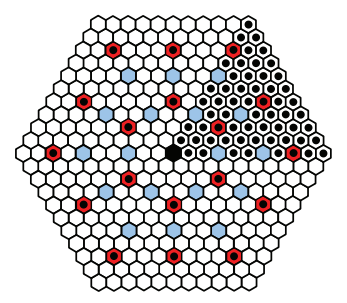

- o Position of measured fuel pin, with Gd

(a)
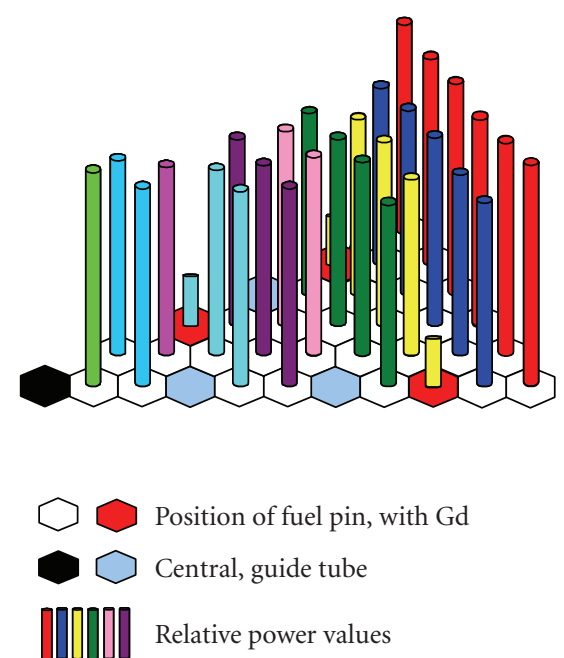

(b)

FIgURe 2: (a) Positions of measured FPs in CFA and (b) power distribution in the 30-degree symmetry sector of the CFA with 3 Gd FPs.

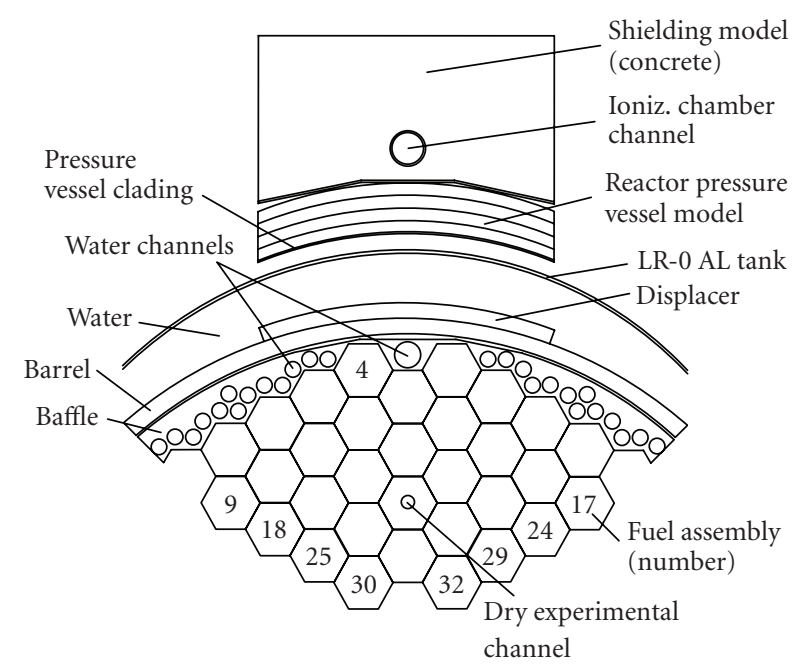

FIgURE 3: The section of the mockup by the XY plane.

were performed in CFA at $74 \mathrm{FPs}$ with positions shown in Figure 2(a); detailed results were presented in [2] and used for evaluation of power distribution in symmetry sector of CFA shown in Figure 2(b). As for this evaluation, absolute value of the difference of power release concerning two FPs in symmetry positions (Figure 2(a)) was calculated and related to the average of these two values and this was done for all above symmetry positions to check reproducibility of the results presented in [2]. The mean and maximum above relative differences are $1.1 \%$ and $3.8 \%$, respectively.

Further experimental results were compared with calculations based on a code treats the two-dimensional, fourgroup diffusion equations using the so-called fine mesh method (each FP cell or absorbing rod cell is considered as one point) and presented in [2]. Results of calculations and measurements are in fairly good agreement: the mean value of the relative power distribution difference absolute values

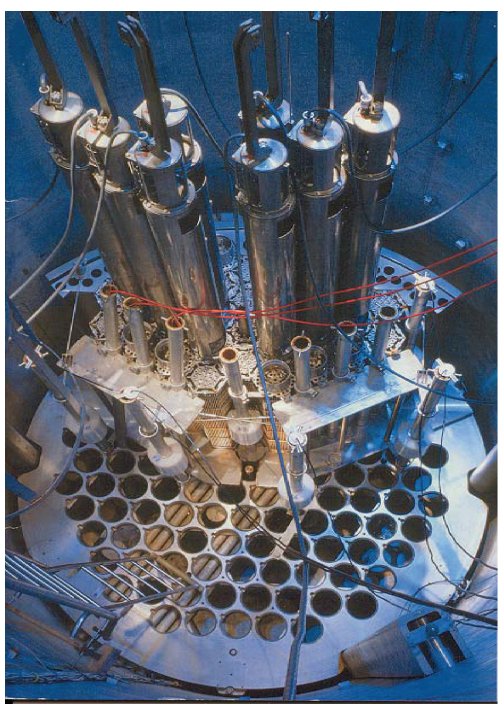

FigURE 4: WWER-1000 mockup general view on reactor LR-0.

is $2.4 \%$; maximum positive and negative relative differences are $6.2 \%$ and $-12.7 \%$, respectively.

Finally the shape of the power distribution in the neighbourhood of a Gd FP can be characterised by the following ratio:

$Q=$ $\frac{\text { (average energy release at ring of } 6 \mathrm{FPs} \text { around a Gd FP) }}{\text { (energy release in that Gd FP) }}$

As for Gd FPs (Figure 1(b)) in positions nos. 1, 2, and 3, the following experimental $Q_{E} /$ calculated $Q_{C}$ values were determined: $3.718 / 3.553,3.669 / 3.501$, and 3.694/3.523 with 


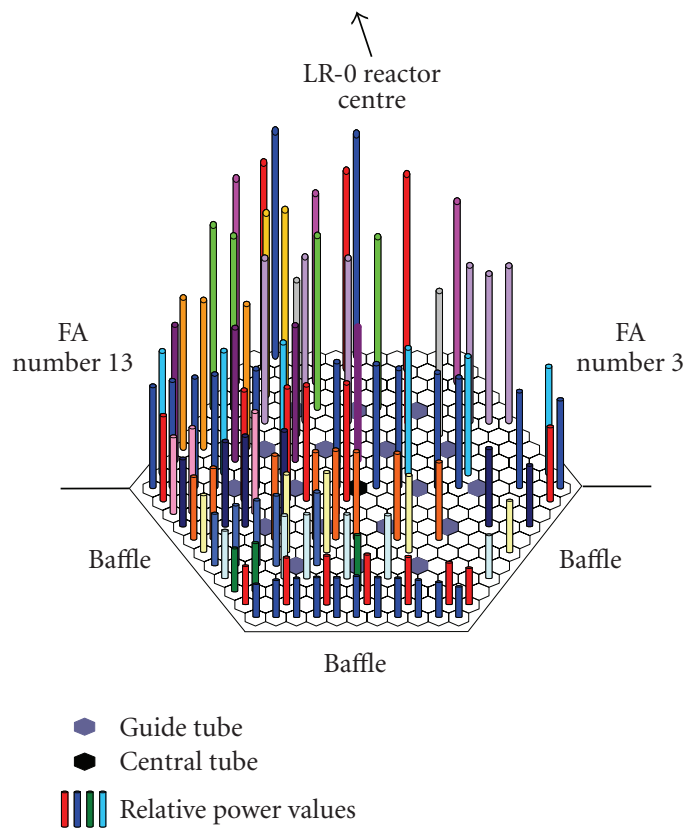

(a)

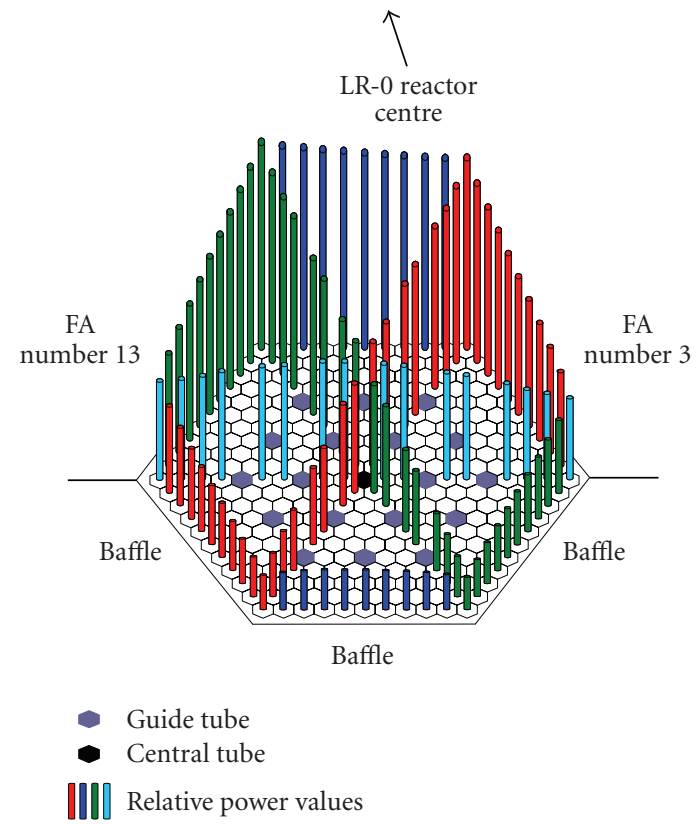

(b)

FIGURE 5: (a) Experimental and (b) calculation power distribution values in selected FP positions in FA no. 4.

relative difference $d=45 \%, 4.7 \%$, and $4.7 \%$, respectively, where

$$
d=\frac{\left(Q_{E}-Q_{C}\right)}{\left[\left(Q_{E}+Q_{C}\right) / 2\right]} .
$$

A fairly good agreement between measured and calculated power distributions can be stated, given by above mean value of $2.4 \%$, as well as between experimental $Q_{E}$ and calculated $Q_{C}$ quantities given by above difference $d$ being about $4.7 \%$ for all three Gd FP positions. The power release depression in Gd FPs can be characterised by the value of $1 / Q$ with corresponding values in positions 1,2 , and 3 being $26.9 \%, 27.3 \%$, and $27.1 \%$, respectively.

\section{INFLUENCE OF BAFFLE IN WWER-1000-TYPE CORE ON POWER DISTRIBUTION IN NEIGHBOURING FA}

\subsection{Experimental arrangement and conditions}

A 60-degree symmetry sector of WWER-1000 mockup in radial direction was realised in the LR- 0 reactor at $4.6 \pm 0.1 \mathrm{~g} / \mathrm{l}$ boron acid concentration in moderator with critical height of $1500 \mathrm{~mm}$ (" 0 " level is at the lower end of FP active part), at atmospheric pressure and room temperature-Figures 3 and 4 .

This mockup represents the core periphery and radial shielding heterogeneities of the WWER-1000. The core loading was chosen to imitate neutron source in $R-\theta$ geometry with following FAs no./enrichment: 2 FAs nos. 9, 17/3.3\%, 6 FAs nos. 18, 24, 25, 29, 30, 32/3.0\% and remaining 24 FAs with $2.0 \%$ enrichment (more information in [3]). The materials published in [3] were used for this work preparation.

\subsection{Results}

Influence of the baffle was investigated by means of power (fission rate) distribution in neighbouring FA no. 4, using gamma scanning of irradiated FPs, measuring their gamma radiation of the ${ }^{140} \mathrm{La}$ with energy $1596.5 \mathrm{keV}$. The radial distribution (in a plane perpendicular to the core axis) was determined by FPs activity of $50 \mathrm{~mm}$ region of FP surface at the central part along rotating FP using corresponding collimator.

Results of the power distribution measurements carried out in $107 \mathrm{FP}$ positions in FA no. 4 are presented in Figure 5(a). Power distribution values were determined with mean and maximum relative errors being $3.2 \%$ and $5 \%$, respectively.

Obtained experimental values were completed by calculations carried out in all $312 \mathrm{FA}$ positions [3]. They are illustrated by values in selected FP positions in Figure 5(b).

Presented results demonstrate depression of the power distribution in FA no. 4 neighbouring baffle that can be characterised by the ratio of the power distribution mean values in two opposite FP rows in FA no. 4 to- and outwards the baffle. The depression, for example, the above ratio of about $19 \%$, can be stated.

\section{DISCUSSION}

As for gadolinium influence, the obtained results have limited information relevance since the experiment was realised in 1990 at special conditions as mentioned above and therefore these results can differ from ones corresponding to the real NPP cores because of dependence, for example, on enrichment and dimensions of the (Gd) FPs, $\mathrm{Gd}_{2} \mathrm{O}_{3}$ 
contents, FP pitch, and Gd FP positions in FA, boron acid concentration in moderator, temperature, pressure, and so forth.

Similar situation can also be stated concerning the baffle influence. Further, a less influence of baffle on power distribution (depression) can be expected in an NPP core (with real WWER-1000 dimension) because of less dimension of LR- 0 core used for investigation.

\section{CONCLUSIONS}

On reactor LR-0 in WWER-1000-type cores the influence of single Gd FPs and the baffle on the space power (fission rate) distribution was investigated. A strong power release depression of about $27 \%$ in a single Gd FP $(3.6 \%$ enrichment, $2.0 \mathrm{wt}$ \% $\mathrm{Gd}_{2} \mathrm{O}_{3}$ ) was determined, given by the ratio of energy release in that Gd FP and average energy release at ring of 6 FPs ( $4.4 \%$ enrichment) around this Gd FP. As for baffle influence in neighbouring FA, the power release depression of about 19\% was estimated, given by the ratio of the power distribution mean values in two opposite FP rows in this FA to- and outwards the baffle.

The above results can be utilised for obtaining some information for the temperature gradients and resulting loads estimation in FPs neighbouring a Gd FP and in FA neighbouring the baffle to consider possible contributions of these loads to the FP failure root causes.

\section{REFERENCES}

[1] J. Mikuš, F. Hudec, J. Roček, M. Trgiňa, L. Vrba, and K. Záleský, "Gadolinium absorbers in WWER-1000 type fuel lattices," in Advanced Calculational Methods for Power Reactors and Lwr Core Design Parameters, Proceedings of the Specialists Meeting, Cadarache, France, September 1990, and a Technical Committee Meeting, pp. 340-344, Řež, Czech Republic, October 1991, IAEA-TECDOC-678.

[2] F. Hudec, J. Mikuš, J. Roček, M. Trgiňa, L. Vrba, and K. Záleský, "Investigations with WWER-1000 type fuel assemblies with Gd-burnable absorbers on LR-0 reactor," in Proceedings of the 1st Symposium of Atomic Energy Research, Řež, Czechoslovakia, September 1991.

[3] B. Ošmera and S. Zaritsky, "WWER-1000 mock-up experiment," Tech. Rep. 36/8-2002, Nuclear Research Institute Řež, Institute of Nuclear Reactors RRC "Kurchatov Institute", Řež, Czech Republic, May 2002, Contract IAEA No. RER 401704583G,

[4] E. A. Panov, Yu. M. Shestakov, and V. N. Miglo, "Analysis of trends in fuel rod depressurization and determination of "gas leak" and "pellet-water interaction" type failures using radiation monitoring techniques of fuel rod leak tightness, fuel failure in normal operation of water reactors: experience, mechanisms and management," in Proceedings of the Technical Committee Meeting, pp. 263-271, Dimitrovgrad, Russia, May 1992, IAEA-TECDOC-709.

[5] Yu. Likhatchev, V. Troyanov, V. Folomeev, and A. Demishonkov, "Theoretical approach to the WWER core thermomechanical modelling," in Proceedings of the 5th International Conference on WWER Fuel Performance, Modelling and Experimental Support, pp. 209-216, Albena, Bulgaria, September-October 2003.
[6] H. G. Weidinger, "PWR and WWER fuel performance: a comparison of major characteristics," in Proceedings of the 6th International Conference on WWER Fuel Performance, Modelling and Experimental Support, pp. 40-50, Albena, Bulgaria, September 2005.

[7] D. Markov, V. Smirnov, A. Smirnov, S. Perepelkin, and V. Polenok, "Integration of post-irradiation examination results of failed WWER fuel rods," in Proceedings of the 5th International Conference on WWER Fuel Performance, Modelling and Experimental Support, pp. 273-277, Albena, Bulgaria, September October 2003. 

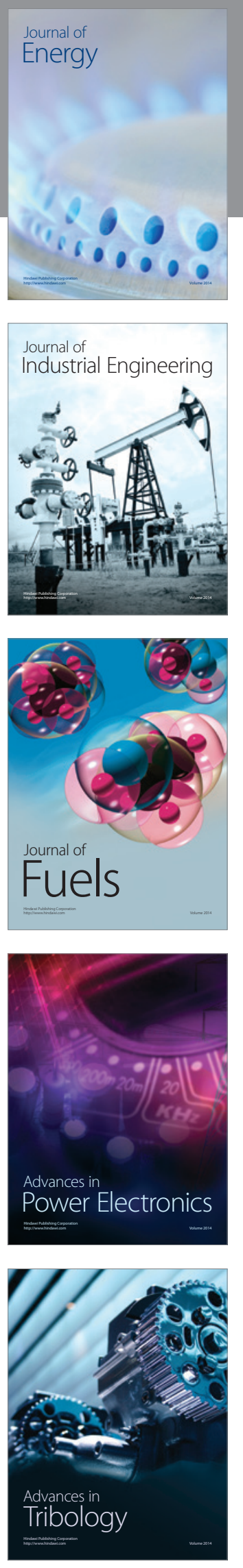
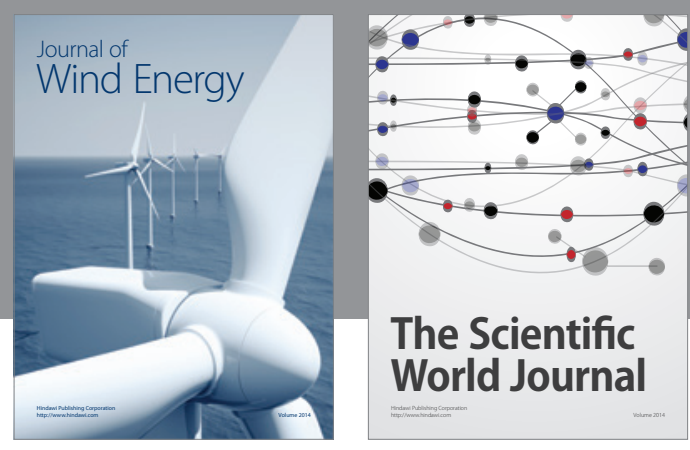

The Scientific World Journal

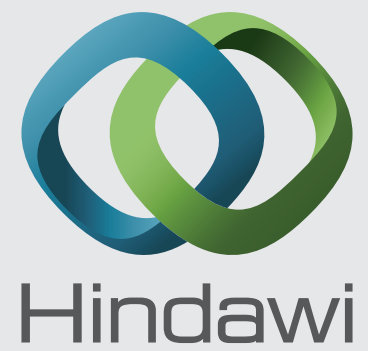

Submit your manuscripts at http://www.hindawi.com
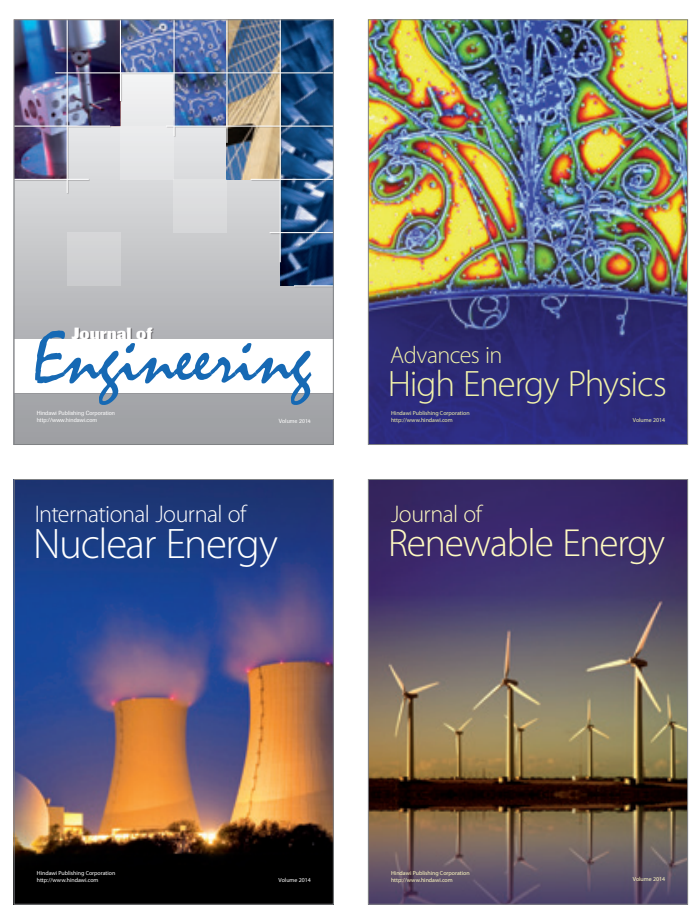

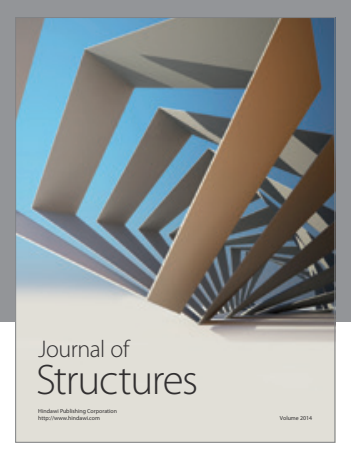

Rotating
Mechinery
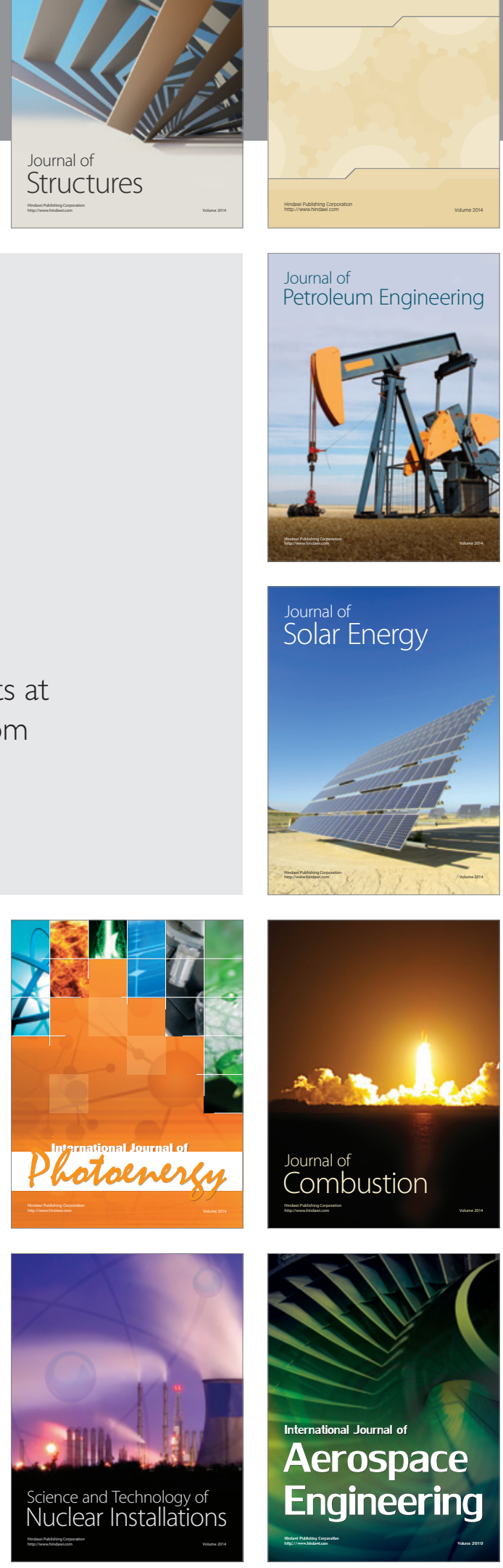